\title{
Human subjective response to steering wheel vibration caused by diesel engine idle
}

\author{
M Ajovalasit and J Giacomin* \\ Department of Mechanical Engineering, The University of Sheffield, Sheffield, UK
}

The manuscript was received on 12 February 2004 and was accepted after revision for publication on 15 October 2004.

DOI: $10.1243 / 095440705 X 11167$

\begin{abstract}
This study investigated the human subjective response to steering wheel vibration of the type caused by a four-cylinder diesel engine idle in passenger cars. Vibrotactile perception was assessed using sinusoidal amplitude-modulated vibratory stimuli of constant energy level (r.m.s. acceleration, $0.41 \mathrm{~m} / \mathrm{s}^{2}$ ) having a carrier frequency of $26 \mathrm{~Hz}$ (i.e. engine firing frequency) and modulation frequency of $6.5 \mathrm{~Hz}$ (half-order engine harmonic). Evaluations of seven levels of modulation depth parameter $m$ $(0.0,0.1,0.2,0.4,0.6,0.8$, and 1.0) were performed in order to define the growth function of human perceived disturbance as a function of amplitude modulation depth. Two semantic descriptors were used (unpleasantness and roughness) and two test methods (the Thurstone paired-comparison method and the Borg CR-10 direct evaluation scale) for a total of four tests. Each test was performed using an independent group of 25 individuals. The results suggest that there is a critical value of modulation depth $m=0.2$ below which human subjects do not perceive differences in amplitude modulation and above which the stimulus-response relationship increases monotonically with a power function. The Stevens power exponents suggest that the perceived unpleasantness is non-linearly dependent on modulation depth $m$ with an exponent greater than 1 and that the perceived roughness is dependent with an exponent close to unity.
\end{abstract}

Keywords: vibration, engine, idle, subjective, perception, modulation

\section{INTRODUCTION}

Amplitude modulation is a commonly observed phenomenon in the vibro-acoustic signatures of many types of mechanical system [1]. Amplitude modulation is present in the vibration measured at the steering wheel of road vehicles at idle due to the rotational irregularity of the engine [2-4]. For a four-cylinder four-stroke engine at idle amplitude modulation occurs because of the action of the halforder engine harmonic on the second-order engine harmonic [5-7]. Comprehensive analytical treatment of the spectral contents of multi-cylinder engines can be found in reference [7]. What follows here is a brief description of the four-cylinder case.

The half-order harmonic of a four-cylinder fourstroke engine arises because there is only a single power stroke occurring in each cylinder every two

* Corresponding author: Department of Mechanical Engineering, The University of Sheffield, Mappin Street, Sheffield S1 3JD, UK. email: m.ajovalasit@sheffield.ac.uk crankshaft revolutions. For an automobile engine the crankshaft rotational speed when at idle is typically in the range from 600 to $840 \mathrm{r} / \mathrm{min}$, corresponding to linear frequencies from 10 to $15 \mathrm{~Hz}$. The half-order harmonic originating from combustion forces in the cylinder is therefore in the range from 5 to $7 \mathrm{~Hz}$, as shown by the experimental measurements performed by Dixon et al. [5].

The second-order $\left(\mathrm{H}_{2}\right)$ and the other even-order harmonics $\left(\mathrm{H}_{4}, \mathrm{H}_{6}\right.$, etc.) are caused instead by mechanical unbalance [6-8]. The reciprocating motion of the pistons and of the connecting rods, combined with the rotational motion of the crankshaft, generate inertial forces that act on the engine block. At low engine speeds the combustion gas forces are greater than the mechanical inertial forces, but at high speeds the opposite is true. In four-cylinder four-stroke engines the first-order inertial forces are normally well balanced since the crankshaft is balanced and the piston pairs move in opposite directions. Vertically acting second-order forces and their multiples are produced, however, because the two descending 
pistons in a four-cylinder engine travel further at a given crankshaft angle than the two ascending pistons since lateral movement of the connecting rods accelerates the descending pistons while delaying the ascending pistons. The centre of gravity of the ascending and descending masses therefore varies, producing a resultant force that varies periodically twice per crankshaft revolution. Laterally acting second-order forces and their multiples also occur due to the angle between the connecting rod and the cylinder. Lateral gas and inertial forces vary periodically twice per crankshaft revolution due to the change in direction of the connecting rod. For an idle speed in the range from 600 to $840 \mathrm{r} / \mathrm{min}$, the corresponding linear second-order linear frequencies are from 20 to $28 \mathrm{~Hz}$.

Amplitude modulation of the form described above is conveniently and compactly represented by means of the modulation depth parameter $m$. This parameter is defined as the amount of change in the amplitude of the waveform, and which is expressed as a proportion by

$$
m=\frac{A_{\max }-A_{\min }}{A_{\max }}
$$

Thus an $m$ value of 1.0 describes an amplitude variation of the carrier sinusoid from zero to a maximum, whereas an $m$ value of 0.0 describes the unmodulated version of the carrier signal (i.e. a pure sine wave). Owing to combustion irregularity an engine tends to run 'rough' and stall much more easily at lower idle speeds [4]. Also, anecdotal evidence suggests that even slight fluctuations of engine idle can cause unpleasant vibrations, leading to lower customer satisfaction. Since smooth idle is an important vehicle attribute perceived by customers [9], identification of an acceptance level of amplitude modulation index $m$ can provide valuable information to vehicle designers.

For the human hand-arm system, considerable psychophysical research has been performed to investigate how the amplitude and the frequency of a vibrotactile stimulus affects detection threshold [10-11] and difference threshold [12]. Results of vibrotactile discrimination tests performed using two superimposed sinusoids at low frequency $(10 \mathrm{~Hz}+$ $30 \mathrm{~Hz})[13]$ presented to the fingertip suggest that the tactile system utilizes a temporal code for amplitude discrimination on the basis of uniformity or non-uniformity of the sequences of perceived peaks within stimulus cycles. Studies of temporal sensitivity in the tactile system performed by Weisemberger [14] defined a modulation transfer function (MTF) that related modulation depth thresholds to the frequency of modulation. MTF can be used to predict the depth of modulation necessary just to allow discrimination between a modulated and an unmodulated waveform. In Weisemberger's experiments the modulated signal was frequently reported as feeling 'rougher' than the unmodulated signal. Lamoré [15] found that high-frequency $(1000-2000 \mathrm{~Hz})$ sinusoidal vibrations of the skin induced maximum sensitivity when amplitude modulation is applied at modulation frequencies between 100 and $300 \mathrm{~Hz}$. The use of high-frequency stimuli makes this an atypical study, being outside the frequency range, up to about $350 \mathrm{~Hz}$ [10], to which the tactile system is maximally responsive. No data regarding the human response to amplitude-modulated vibration of the type caused by engine idle are available in the scientific literature.

Psychophysical methods developed for the scaling of sensory attributes such as perceived roughness [16] or perceived intensity [17] can measure human subjective response to many forms of non-metric (i.e. stimuli that can be arranged only on a nominal scale) and metric stimuli. The end objective of such tests is the establishment of some form of metric scale that describes the relationship between the physical properties of the stimulus and the subjective characteristics of the human response. In many applications the relationship can be compactly summarized by means of the well-known Stevens power law that relates human response to numerous environmental stimuli. The psychophysical test protocols themselves can be divided into two major classes: those involving indirect rating of the stimuli by means of paired comparisons and those in which a test subject directly provides an estimate of his or her response by means of a fixed scale. Unfortunately, the scientific literature provides little conclusive evidence $[\mathbf{1 8}, \mathbf{1 9}]$ of the superiority of one protocol with respect to the other. It is therefore good practice to evaluate the potential differences that can occur due to the choice of psychophysical test protocol.

This paper presents an investigation of the growth in the human subjective response to amplitudemodulated steering wheel idle vibration stimuli as a function of the modulation depth $m$. Both an indirect and a direct scaling method have been used to assess stimuli under two sensory attributes (unpleasantness and roughness) in order to evaluate possible differences. Psychophysical response scales were constructed by means of the Thurstone law of comparative judgement [20], which provided an indirect scaling method, and the category ratio Borg CR-10 scale [21], which is a direct scaling method. 


\section{TESTING OF HUMAN RESPONSE TO AMPLITUDE-MODULATED STEERING WHEEL VIBRATION STIMULI}

\subsection{Experimental apparatus}

A test facility for applying rotational vibration to a seated test subject was used in this study. A schematic representation of the steering wheel test rig and associated data-conditioning and acquisition systems are shown in Fig. 1.

The rotational steering system consists of an aluminium wheel of $325 \mathrm{~mm}$ diameter connected to a steel shaft which is mounted onto two high precision bearings. The shaft is connected to a G\&W V20 electrodynamic shaker and associated PA 100 power amplifier by means of a steel stinger rod. Bench control and data acquisition were performed by means of LMS Cada-X 3.5 E software and a 12-channel Difa Systems Scadas III front-end unit. The acceleration obtained at the steering wheel was measured using an Entran EGAS-FS-25 accelerometer located on the top left side of the wheel. The acceleration was measured in the tangential direction. The accelerometer signal was amplified by means of an Entran MSC6 signal-conditioning unit. Table 1 presents the rig main geometric dimensions, which were chosen on the basis of data from a small European auto-

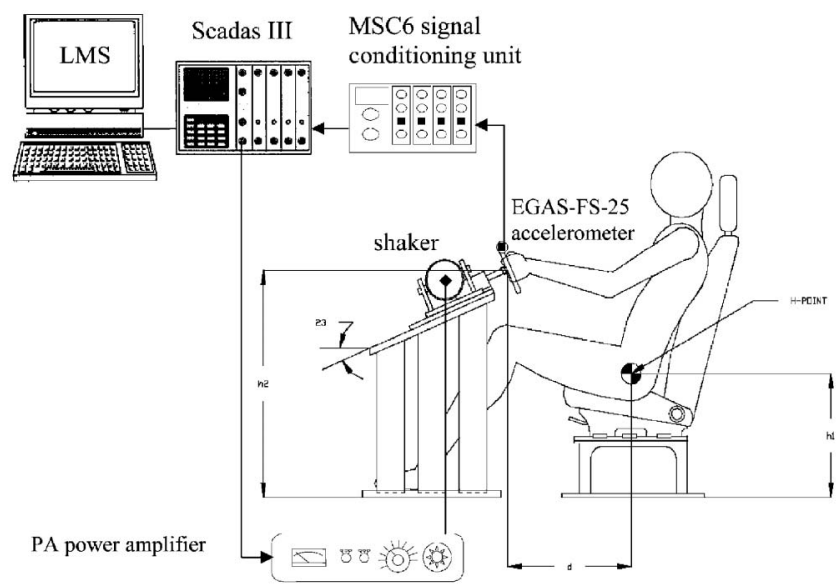

Fig. 1 Steering wheel rotational vibration test rig and associated electronics mobile. The seat, taken from a small European automobile, was fully adjustable in terms of horizontal travel and backrest inclination.

The test bench had a first resonance frequency of $350 \mathrm{~Hz}$. When loaded by a human hand-arm system and tested using sinusoidal excitation at frequencies of 4.0, 8.0, 16.0, 31.5, 63.0, 125, and $250 \mathrm{~Hz}$ at amplitudes of $0.2,2.0$, and $20.0 \mathrm{~m} / \mathrm{s}^{2}$ r.m.s. the bench provided a maximum total harmonic distortion (THD) of 15 per cent at $4 \mathrm{~Hz}$ and $20 \mathrm{~m} / \mathrm{s}^{2}$ [22]. With both increasing frequency and decreasing amplitude the THD dropped to a minimum of 0.002 per cent at $250 \mathrm{~Hz}$ and $0.2 \mathrm{~m} / \mathrm{s}^{2}$. During the same tests, a linear fore-and-aft direction acceleration measurement was also performed at the same point on the rigid wheel. Fore-and-aft acceleration was found to be no greater than $-50 \mathrm{~dB}$ with respect to the tangential acceleration in all cases measured.

Pretesting using amplitude-modulated test stimuli showed that the dynamic response of the test bench produced unequal harmonic side bands due to the frequency response of the shaker. Compensated drive voltage signals were therefore defined which included the effect of shaker frequency response. Figure 2 presents the power spectral density (PSD) of the target test stimuli and of the bench response signals at a modulation depth $m=1.0$ for both the uncompensated (Fig. 2a) and the compensated (Fig. 2b) drive voltage signals. With frequency compensation the modulated acceleration stimuli were reproduced at the human test subject with r.m.s. errors of less than 5 per cent.

\subsection{Test signals}

Engine idle vibration occurring at the steering wheel of road vehicles was simulated by means of amplitude-modulated acceleration time histories. Sinusoidal carriers were chosen as the best reproduction of the steering wheel idle vibration signals based on previous research by the present authors [2] The instantaneous amplitude $A(t)$ of a sinusoidal amplitude-modulated signal is given by

$$
A(t)=A_{0}\left[1+m \sin \left(2 \pi f_{\mathrm{m}} t+\varphi\right)\right] \sin \left(2 \pi f_{\mathrm{c}} t+\theta\right)
$$

Table 1 Geometric dimensions of the steering wheel rotational vibration test rig

\begin{tabular}{ll}
\hline Geometric parameter & Value \\
\hline Seat H point height from floor, $h_{1}$ & $275 \mathrm{~mm}$ \\
Horizontal distance adjustable from H point to steering wheel hub centre, $d$ & $390-550 \mathrm{~mm}$ \\
Steering wheel hub centre height above floor, $h_{2}$ & $710 \mathrm{~mm}$ \\
Steering column angle with respect to floor & $23^{\circ}$ \\
Steering wheel handle diameter & $12.5 \mathrm{~mm}$ \\
Steering wheel diameter & $325 \mathrm{~mm}$ \\
\hline
\end{tabular}



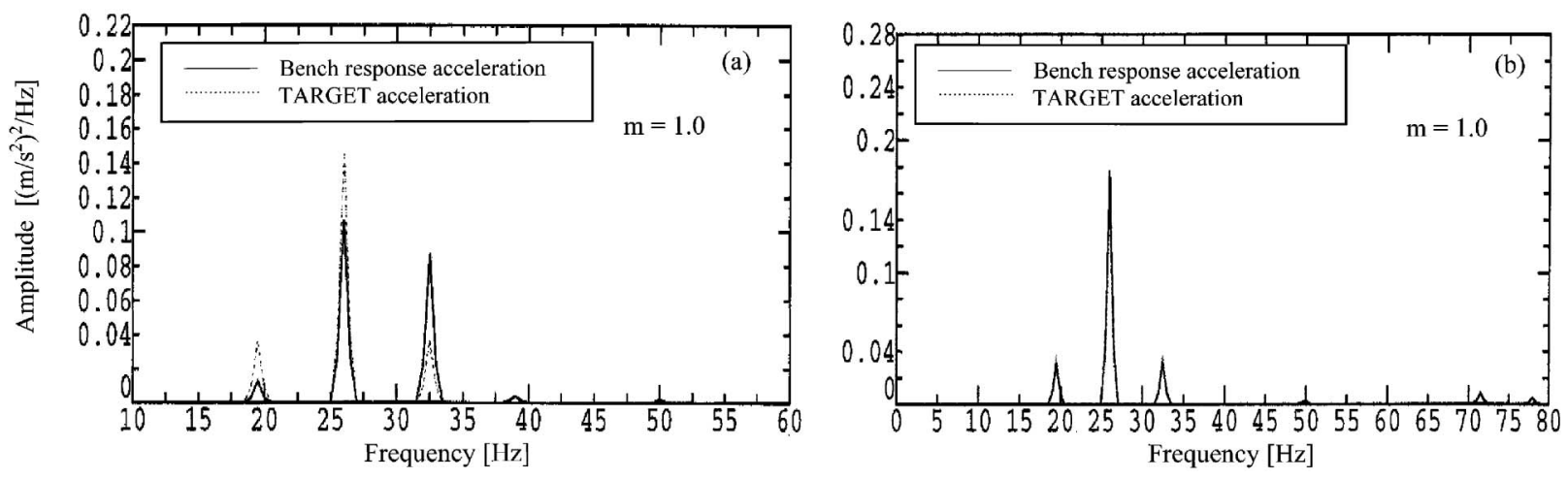

Fig. 2 Test signals: (a) comparison between target and bench response acceleration before compensation; (b) comparison between target and bench response acceleration after compensation

where $A_{0}$ is the amplitude of the carrier signal, $m$ is the modulation depth, $f_{\mathrm{m}}$ is the modulation frequency, $f_{\mathrm{c}}$ is the carrier frequency, $t$ is a time increment, and $\varphi$ and $\theta$ are the phases of the modulating and carrier signals respectively. In all the experiments presented here the phases of both the carrier and the modulating waves were chosen to be equal to zero for simplicity $(\varphi=0$ and $\theta=0)$. The frequency spectrum of the amplitude modulated waveform described by equation (2) consists of a component at the carrier frequency, and modulation components (side bands) that are above and below the carrier.
For a four-cylinder four-stroke diesel engine idle at $780 \mathrm{r} / \mathrm{min}$ [2] the firing frequency harmonic of $26 \mathrm{~Hz}$ can be considered as the carrier frequency $f_{\mathrm{c}}$ and a half-order of $6.5 \mathrm{~Hz}$ can be considered as the modulation frequency $f_{\mathrm{m}}$. Figure 3 presents two steering wheel idle vibration signatures measured along the vertical $(z)$ direction in terms of both the time history and its relative PSD for two different fuel conditions. The spectra, obtained using a Hanning window and a frequency resolution of $0.5 \mathrm{~Hz}$, illustrate how the energy conveyed to the steering wheel normally covers only the low-frequency range up to
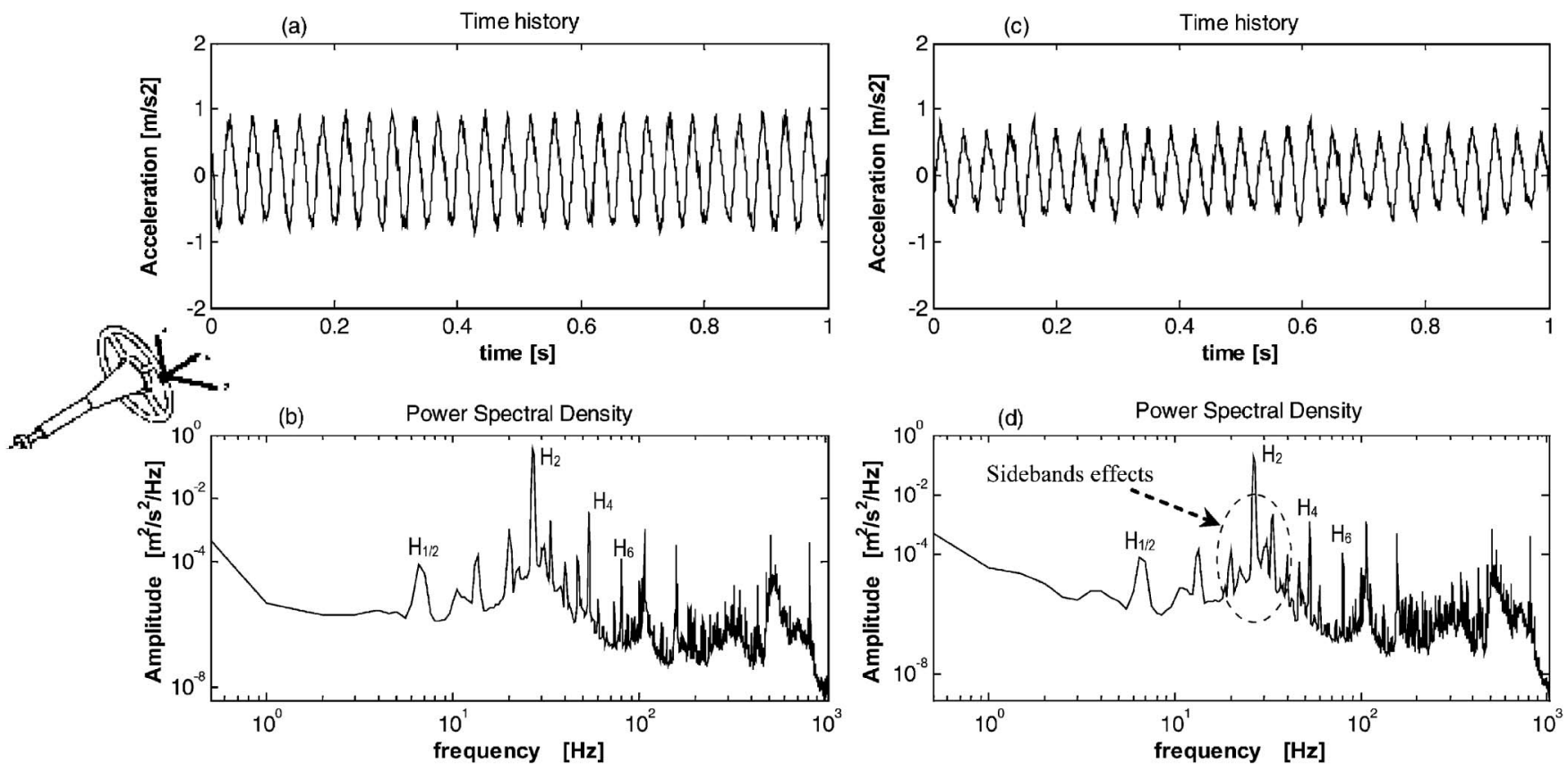

Fig. 3 Steering wheel idle vibration signature along the vertical $(z)$ axis for the weakly modulated condition [(a) time history and (b) its relative PSD] and the strongly modulated condition [(c) time history and (d) its relative PSD] 
$200 \mathrm{~Hz}$. The second harmonic $\mathrm{H}_{2}$ (at about $26 \mathrm{~Hz}$ ) and its modulation side bands can be seen to be prominent as expected for a four-cylinder fourstroke engine [5-7]. With respect to similar measurements performed at the engine itself, the even-order harmonics $\mathrm{H}_{4}(52 \mathrm{~Hz}), \mathrm{H}_{6}(78 \mathrm{~Hz})$, and higher orders are attenuated in the path through the mechanical component of steering system. Test results from a fuel having a higher cetane number (52.9) are presented in Figs $3 a$ and $b$ while results from a fuel having a lower cetane number (44.7) are presented in Figs $3 c$ and d. Comparison of both the time histories and the PSD suggests that the amplitude modulation of the firing frequency is characteristic of deteriorated fuel conditions.

In the present experiment the test stimuli consisted of amplitude modulated sinusoids with a $26 \mathrm{~Hz}$ carrier frequency and a modulation frequency of $6.5 \mathrm{~Hz}$, producing side band components at 19.5 and $32.5 \mathrm{~Hz}$. A sampling rate of $512 \mathrm{~Hz}$ was chosen to generate the signals since the typical range of engine idle speeds of road vehicles is from 600 to $840 \mathrm{r} / \mathrm{min}$, leading to firing frequencies ranging from 20 to $28 \mathrm{~Hz}$ and higher harmonics up to $100 \mathrm{~Hz}$. Seven different values of modulation depth parameter $m$ equal to $0.0,0.1,0.2,0.4,0.6,0.8$, and 1.0 were chosen. Since the average power of a signal is increased when the carrier is modulated [23], all the seven stimuli were scaled to have equal average power in order to eliminate changes in overall perceived intensity with changes in modulation depth. Average power was quantified by means of r.m.s. acceleration $a_{\text {r.m.s. }}$ defined by

$$
a_{\text {r.m.s. }}=\left[\frac{1}{T} \int_{0}^{T} a^{2}(t) \mathrm{d} t\right]^{1 / 2}
$$

where $T$ is the duration over which the r.m.s. acceleration is measured, $a(t)$ is the acceleration value and $t$ is a time increment. The reference r.m.s. acceleration chosen for all stimuli was the average r.m.s. value measured from the steering wheel idle vibration time histories recorded from a Ford Focus test vehicle for 12 different fuel conditions [2]. The r.m.s. accelerations for the 12 fuel conditions ranged from 0.31 to $0.43 \mathrm{~m} / \mathrm{s}^{2}$ with an average value of $0.41 \mathrm{~m} / \mathrm{s}^{2}$. Thus the value of the amplitude of the test carrier signal was calculated to be $A_{0}=\sqrt{2} a_{\text {r.m.s. }}=$ $\sqrt{2} \times 0.41=0.58 \mathrm{~m} / \mathrm{s}^{2}$. The duration of each test signal was chosen to be $4 \mathrm{~s}$ based on the knowledge that the tactile system of the hand does not present temporal integration properties below approximately $40 \mathrm{~Hz}$ [24] and based on the results of experiments reported by Miwa [25] who suggested that for vibration in the range $2-60 \mathrm{~Hz}$ there may be no further increase in discomfort sensation for stimuli durations greater than approximately $2 \mathrm{~s}$.

In addition to the r.m.s. acceleration, the vibration dose value (VDV) was also calculated for all the seven signals by integrating the fourth power of the acceleration $A(t)$ of equation (2) as defined in BS 6841 [26] and this is given by

$$
\mathrm{VDV}=\left[\int_{0}^{T} A^{4}(t) \mathrm{d} t\right]^{1 / 4}
$$

where $T$ is the duration over which the VDV is measured and $A(t)$ is the instantaneous acceleration amplitude of the signal. The VDV provides a cumulative measure of the vibration exposure, and being a fourth-power method it accounts more accurately for the greater effect on human response to vibration of high-amplitude peaks that occur in the time history [27]. Figure 4 presents the seven time history test signals used in all experiments.

\subsection{Test methodology}

Four experimental conditions, determined by the combination of two subjective descriptors and two test methods, were conducted to examine the human subjective response to steering wheel idle vibration. The semantic attributes of unpleasantness and roughness were chosen as descriptors of the human response to idle vibration since they have been found to be used by drivers to describe vehicle idle quality [9]. In addition the sensory attribute of roughness was also chosen based on the feeling 'rougher' reported in Weisenberger's experiments [14]. Experiments I and II used the scaling method according to the Thurstone law of comparative judgement (case III). Experiments III and IV used the category ratio Borg CR-10 scale under the same sensory attributes of perceived unpleasantness and perceived roughness.

In experiments I and II all possible pairings of the seven test signals were used in order to counterbalance the test fully [28]. The result was a total of 42 paired comparisons. In order to reduce testing time no stimulus was presented with its duplicate as a pair. Each subjective evaluation consisted of a $4 \mathrm{~s}$ test signal followed by a $2 \mathrm{~s}$ gap followed by the other $4 \mathrm{~s}$ test signal. Each paired comparison therefore required $10 \mathrm{~s}$, a duration which was chosen to keep the stimulus short enough to permit both signals to remain in the human short-term memory for tactile stimuli [29]. To reduce learning and fatigue effects the order of presentation of the 42 comparisons was randomized for each test subject. After presenting 


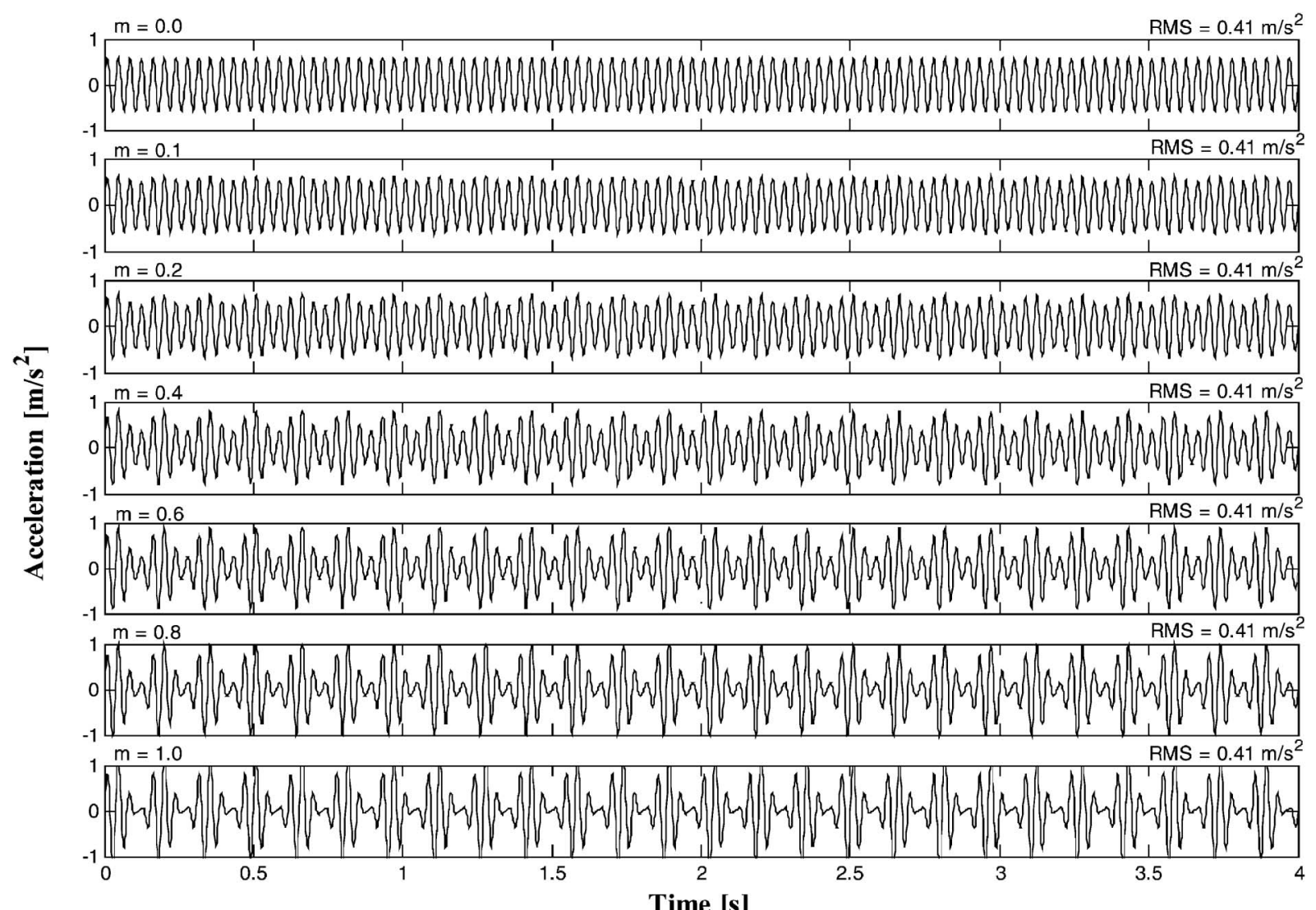

Time [s]

Fig. 4 Test signals used in all experiments (RMS indicates r.m.s. acceleration value)

each stimuli pair the test subject was asked to indicate which stimuli they considered to be 'more unpleasant' in experiment I or the 'rougher' in experiment II. The complete paired-comparison test (all 42 pairs) lasted 16 min for each test subject.

In experiments III and IV perceived unpleasantness and perceived roughness of the vibration were assessed using the Borg CR-10 scale following the instructions provided by Borg [21] for the scale's administration. The Borg CR-10 scale (shown in Fig. 5) consists of a numerical scale from 0 (nothing at all) to 10 (extremely strong) with nine verbal anchors placed along the scale in an approximately logarithmic fashion. The test subjects were asked to judge each test stimuli on its own merits, independent of preceding stimuli. At the start of testing a familiarization period was used to acquaint the subject with the use of the scale by means of practice ratings using a non-vibrational stimuli (acidity rating of common foods) and a dummy test involving two vibrational stimuli selected from the test set. The same seven signals used in the experiments I and II were employed for the direct Borg CR-10 scaling

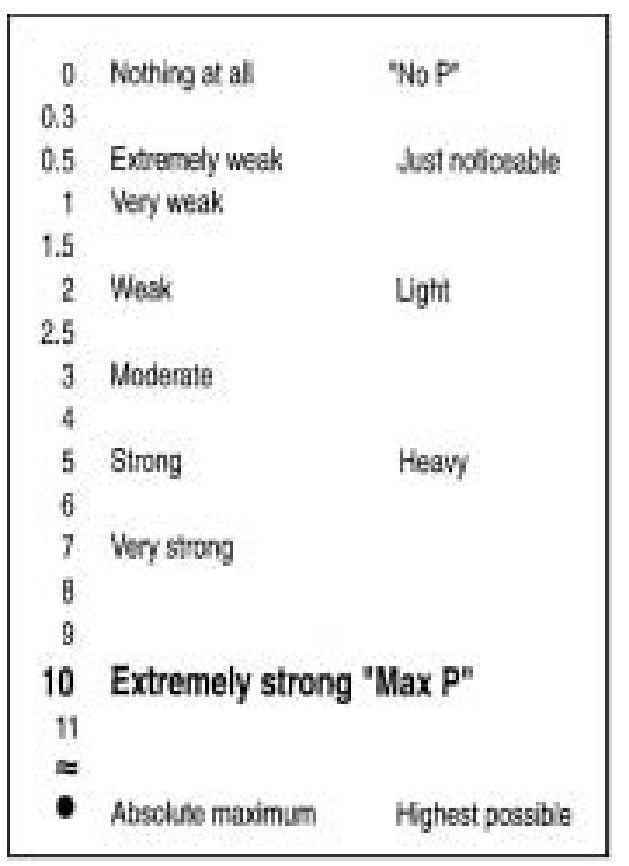

Fig. 5 Borg CR-10 scale. (Adapted from Borg [8]) 
method. All the stimuli had the same time duration of $4 \mathrm{~s}$ as in the paired comparison tests. In order to assess the individual's ability to rate stimuli using the Borg scale each of the seven exposures was repeated four times, giving a total of 28 assessment trials. In order to minimize any possible bias resulting from learning or fatigue effects the order of presentation of the test signals was randomized for each subject. A break of $10 \mathrm{~s}$ after the presentation of each set and a break of $5 \mathrm{~s}$ between each trial were allowed to avoid annoyance effects. Total testing time for a single test subject amounted to $13 \mathrm{~min}$.

\subsection{Test subjects and test protocol}

An independent group of 25 individuals was tested for each of the four test conditions. Upon arrival in the laboratory each participant was given an information sheet and a consent form describing the purpose, procedure, risks, and time commitment for the research project. After providing written consent, subjects were given a verbal description of the experiment and a short questionnaire regarding their physical characteristics, health, driving experience, and history of previous vibration exposure. The test groups consisted of University of Sheffield students and staff, whose age, height, and weight characteristics are summarized in Table 2. On average, 88 per cent of the subjects drove $2-10 \mathrm{~h}$ daily and all declared that they were in good physical and mental condition. Before commencing testing, each subject was asked to remove any coats, watches, or jewellery and then to adjust the seat position and backrest angle so as to simulate a driving task as realistically as possible. Subjects were required to maintain a constant palm grip [30] on the steering wheel using both hands, as when driving on a winding country road. They were asked to wear ear protectors to avoid audio cues. During the paired-comparison experiment they were also asked to wear opaque glasses and to close their eyes to avoid any visual distractions, whereas during the Borg CR-10 scale experiments they were encouraged to focus their eyes on a placard representing the perceived rating Borg scale placed about $1 \mathrm{~m}$ ahead at eye level. Room temperature was maintained in the range from 20 to $25^{\circ} \mathrm{C}$ so as to avoid significant environmental effects on the skin sensitivity [31]. The test facility and test protocol were reviewed and found to meet the University of Sheffield guidelines for good research practice.

\section{RESULTS}

From the average test results for each test group of 25 subjects an interval scale was constructed according to Thurstone's comparative judgement from experiments I and II, and a ratio scale was constructed according to the direct scaling method using the Borg CR-10 scale from experiments III and IV. The mean scale values obtained for each value of modulation depth in each of the four experiments, together with the standard deviation (SD) values, are provided in Table 3. The Thurstone method produces as output a scale difference between the means of the stimulus responses, locating the stimuli on the interval scale with respect to one another. Direct rating by means of the Borg CR-10 scale produces a subjective value for each stimulus placing it along a scale that is claimed to have ratio scale properties. The mean

Table 2 Physical characteristics of the four groups of test subjects

\begin{tabular}{|c|c|c|c|c|}
\hline & & $\begin{array}{l}\text { Age } \\
\text { (years) }\end{array}$ & $\begin{array}{l}\text { Height } \\
(\mathrm{cm})\end{array}$ & $\begin{array}{l}\text { Mass } \\
(\mathrm{kg})\end{array}$ \\
\hline \multicolumn{5}{|l|}{ Group I $(n=25)$} \\
\hline Perceived & Mean (SD*) & $27.4(7.93)$ & $1.7(0.08)$ & $70.4(14.10)$ \\
\hline unpleasantness & Minimum & 20.0 & 160.0 & 45.0 \\
\hline (Pair comparison) & Maximum & 56.0 & 190.0 & 100.0 \\
\hline \multicolumn{5}{|l|}{ Group II $(n=25)$} \\
\hline Perceived & Mean (SD*) & $29.3(5.12)$ & $1.7(0.09)$ & 74.1 (16.39) \\
\hline roughness & Minimum & 22.0 & 160.0 & 48.0 \\
\hline (Pair comparison) & Maximum & 41.0 & 188.0 & 111.2 \\
\hline \multicolumn{5}{|l|}{ Group III $(n=25)$} \\
\hline Perceived & Mean (SD*) & $28.5(5.04)$ & $1.7(0.08)$ & $75.8(14.30)$ \\
\hline unpleasantness & Minimum & 22.0 & 160.0 & 53.0 \\
\hline (Borg CR10 scale) & Maximum & 42.0 & 185.0 & 107.0 \\
\hline \multicolumn{5}{|l|}{ Group IV $(n=25)$} \\
\hline Perceived & Mean (SD*) & $29.4(6.55)$ & $1.8(0.107)$ & $76.0(15.69)$ \\
\hline roughness & Minimum & 22.0 & 160.0 & 50.0 \\
\hline (Borg CR10 scale) & Maximum & 48.0 & 201.0 & 115.8 \\
\hline
\end{tabular}


Table 3 Subjective raw scale mean values and in parentheses, SDs for the seven test stimuli

\begin{tabular}{|c|c|c|c|c|c|c|}
\hline \multicolumn{3}{|c|}{ Stimuli } & \multicolumn{2}{|c|}{ Thurstone scale values } & \multicolumn{2}{|c|}{ Borg scale values } \\
\hline $\begin{array}{l}\text { Modulation } \\
\text { depth } m\end{array}$ & $\begin{array}{l}a_{\text {r.m.s. }} \\
\left(\mathrm{m} / \mathrm{s}^{2}\right)\end{array}$ & $\begin{array}{l}\text { VDV } \\
\left(\mathrm{m} / \mathrm{s}^{1.75}\right)\end{array}$ & $\begin{array}{l}\text { Experiment I: } \\
\text { relative unpleasantness }\end{array}$ & $\begin{array}{l}\text { Experiment II: } \\
\text { relative roughness }\end{array}$ & $\begin{array}{l}\text { Experiment III: } \\
\text { perceived unpleasantness }\end{array}$ & $\begin{array}{l}\text { Experiment IV: } \\
\text { perceived roughness }\end{array}$ \\
\hline 0.0 & 0.41 & 0.640 & $0 \quad(0.338)$ & $0 \quad(0.982)$ & $2.38(1.212)$ & $2.45(0.708)$ \\
\hline 0.1 & 0.41 & 0.644 & $-0.302(0.288)$ & $-0.054(0.913)$ & $2.42(1.087)$ & $2.75(0.792)$ \\
\hline 0.2 & 0.41 & 0.658 & $-0.077(0.386)$ & 0.287 (1.098) & $2.48(1.080)$ & $2.78(0.915)$ \\
\hline 0.4 & 0.41 & 0.707 & $0.3 \quad(0.414)$ & $1.113(1.419)$ & $3.06(1.225)$ & $3.37(0.973)$ \\
\hline 0.6 & 0.41 & 0.773 & $0.895(0.343)$ & $1.853(1.256)$ & $3.44(1.398)$ & $4.03(1.186)$ \\
\hline 0.8 & 0.41 & 0.847 & $1.16 \quad(0.357)$ & $2.588(0.795)$ & 3.79 (1.638) & $4.22(1.412)$ \\
\hline 1.0 & 0.41 & 0.925 & $2.302(0.182)$ & $3.555(0.587)$ & $3.93(1.628)$ & $4.55(1.582)$ \\
\hline
\end{tabular}

scale values and the standard deviation values are presented for each of the four experiments in Fig. 6.

Since the zero point of the Thurstone interval scale and the unit of measurement of the Borg ratio scales are not unique, a common scale was required to facilitate comparisons. Studies of the temporal sensitivity of the tactile system performed by Weisenberger [14] indicated that the modulation
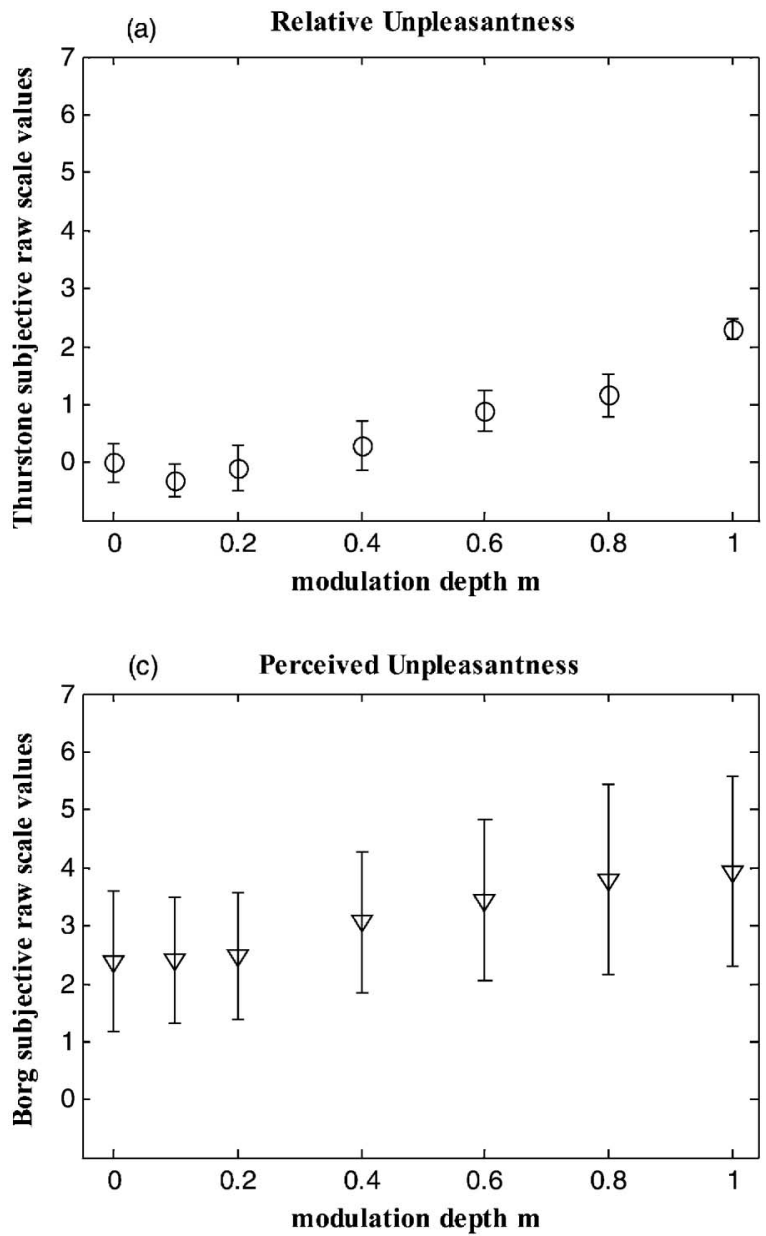

depth $m_{\text {th }}$ necessary just to allow discrimination between a modulated and an unmodulated sinusoidal carrier waveform of $25 \mathrm{~Hz}$ was 0.2 for modulation frequencies ranging from 5 to $10 \mathrm{~Hz}$. Based on this observation, and the current results for the experimental condition of $f=26 \mathrm{~Hz}$ and $f=6.5 \mathrm{~Hz}$ (as shown in Fig. 6), it can be assumed that a modulation depth value of approximately 0.2 represents a point
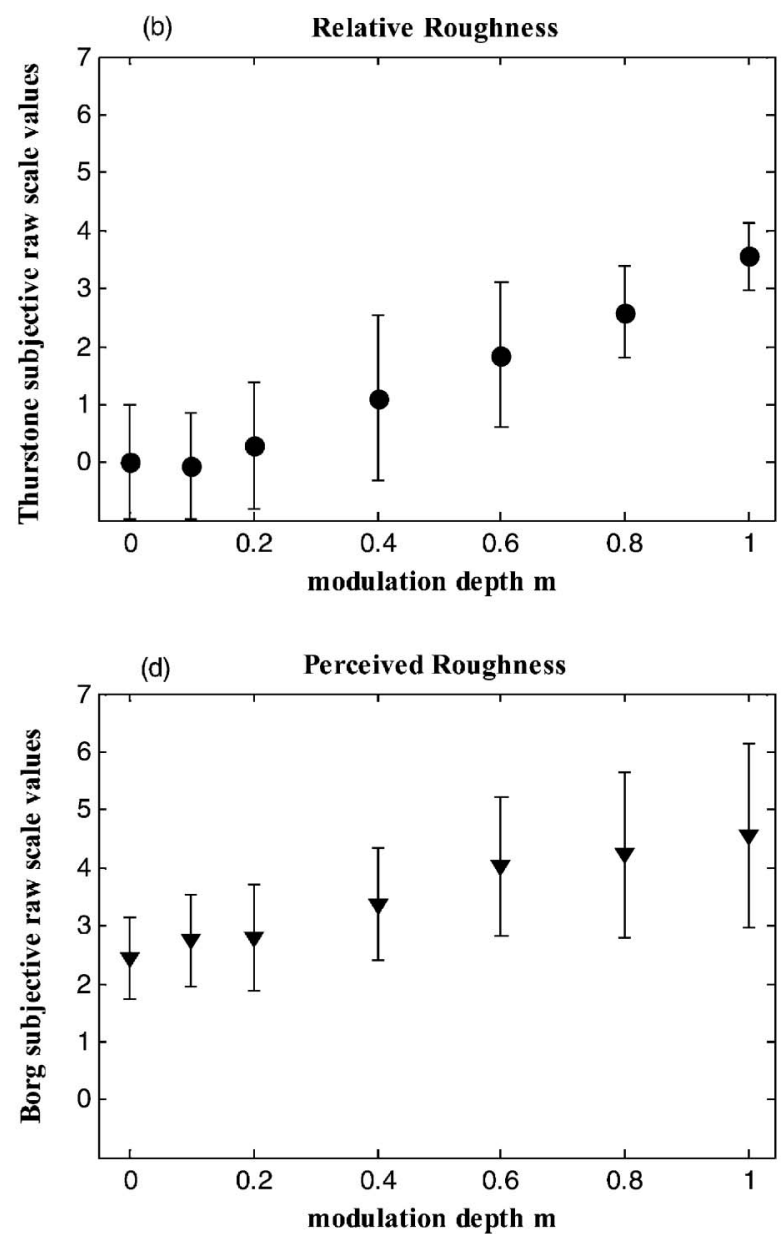

Fig. 6 Subjective raw scale values as a function of modulation depth $m$ : (a) relative unpleasantness, Thurstone scale; (b) relative roughness, Thurstone scale; (c) perceived unpleasantness, Borg CR-10 scale; (d) perceived roughness, Borg CR-10 scale. Data are shown as mean $\pm 1 \mathrm{SD}$ 
of separation between two different human response characteristics. Below the point $m=0.2$, subjects do not perceive differences in amplitude modulation and the sensation magnitude can be interpreted as sensory noise relative to the energy of the unmodulated waveform. Above the point $m=0.2$ the sensation magnitude growths monotonically as a function of the modulation depth $m$. A schematic representation of the proposed model of human perception of amplitude-modulated vibration delivered to the hand is shown in Fig. 7.

Psychophysical relations of the type occurring for modulation depths greater than 0.2 are compactly expressed by means of the well-known Stevens power law which in its most general form is expressed as

$$
R=R_{0}+k\left(X-X_{\text {th }}\right)^{n}
$$

where $R$ is the subjective perceived magnitude, $X$ is the stimulus magnitude (expressed here as the modulation depth $m$ ), $k$ is a constant determined from the measurement units, $n$ is the exponent of the power law, and the two constants $R_{0}$ and $X_{\mathrm{th}}$ indicate the starting point of the growth function on the response axis ( $y$ axis) and on the stimulus axis ( $x$ axis) respectively. In the current analysis the constant $R_{0}$ represents the sensory response to the harmonic stimuli when no actual modulation is present and $X_{\mathrm{th}}$ is the value of the modulation depth at threshold $m_{\text {th }}$. The use of equation (5) implies that the sensation magnitude $R$ is a power function of effective stimulation above the threshold $m_{\mathrm{th}}$ of amplitude modulation. The value $X_{\text {th }}$ to which the physical stimuli on the $x$ axis were rescaled was chosen equal to $m_{\mathrm{th}}=0.2$, whereas the constant value $R_{0}$ was taken to be equal to the average value of the responses to the stimulus corresponding to modulation depths $m=0.0, m=0.1$, and $m=0.2$ for both psychophysical scales.
After translating the raw data on both axes the growth functions of human-perceived unpleasantness and roughness of the vibration could be determined as a function of the difference in modulation depth with respect to threshold, $m^{*}=m-m_{\mathrm{th}}$, as shown in Fig. 8. Figure 8a presents the experimentally obtained unpleasantness scale values and the fitted Stevens power law obtained using the Thurstone method $(n=1.38)$ and obtained using the Borg CR-10 scale $(n=0.61)$. Figure $8 \mathrm{~b}$ presents the roughness scale values and the Stevens power law obtained using the Thurstone method $(n=0.92)$ and obtained using the Borg CR-10 scale $(n=0.63)$. In order to test the internal consistency of the Thurstone scale values, a $\chi^{2}[\mathbf{2 8}]$ was used. No systematic deviations between the Thurstone scale values were found at significance level $p<0.01$. In order to identify any statistically significant differences among the Borg scale values, a one-factor analysis of variance test was performed using the modulation depth parameter $m$ as the independent variable. Statistically significant differences between the Borg scale values were found at 1 per cent confidence level $(p<0.01)$. For each of the four experiments the coefficient of determination, $R^{2}$, was also determined (Table 4 ) when correlating the subjective responses to the modulation index $m^{*}$.

\section{DISCUSSION}

The sensory attributes of vibration unpleasantness and vibration roughness belong to the group of perceptual dimensions based on the quality, rather than on the intensity, of the stimulus. Such dimensions are generally thought to depend on more than a single sense modality [32]. Studies performed for this class of sensory continua have shown that subjective

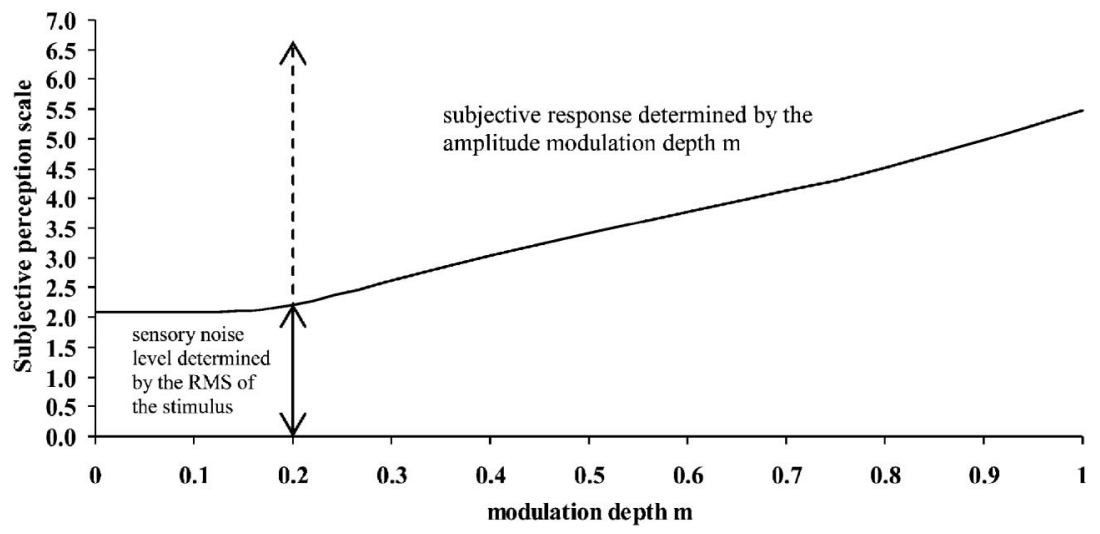

Fig. 7 Model of human hand-arm perception of vibrotactile amplitude-modulated stimuli 

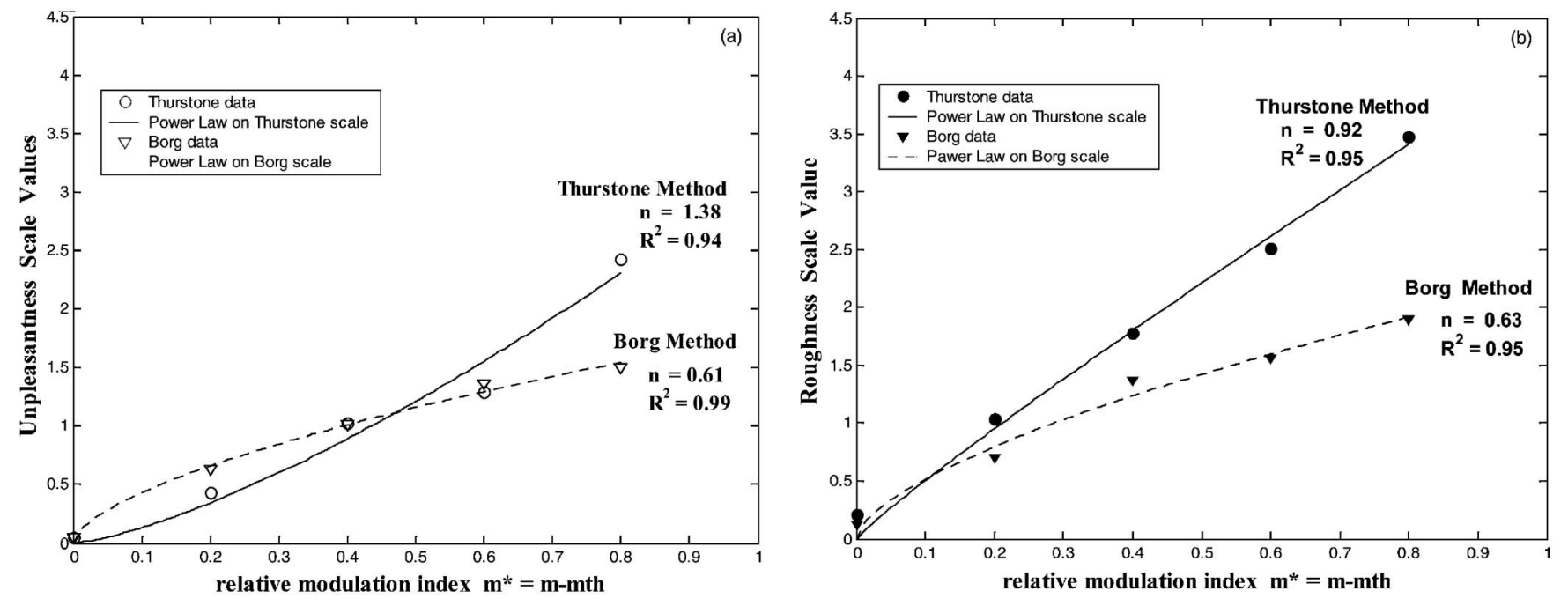

Fig. 8 Growth functions of human perceived disturbance of amplitude-modulated steering wheel idle vibration obtained by means of the Thurstone paired comparison method and by using the Borg CR-10 scale: (a) perceived unpleasantness; (b) perceived roughness

Table 4 The Stevens power exponents $n$ and coefficients of determination, $R^{2}$, determined from the data from experiments I, II, III, and IV

\begin{tabular}{lllll}
\hline Experiment & Scaling method & Sensor attribute & $\begin{array}{l}\text { Stevens power } \\
\text { exponent, } n\end{array}$ & $\begin{array}{l}\text { Coefficient of } \\
\text { determination, } R^{2 *}\end{array}$ \\
\hline I & Thurstone & Perceived unpleasantness & 1.38 & 0.94 \\
II & $\quad$ (indirect method) & Perceived roughness & 0.92 & 0.95 \\
III & Borg CR-10 scale & Perceived unpleasantness & 0.61 & 0.99 \\
IV & (direct method) & Perceived roughness & 0.63 & 0.95 \\
\hline
\end{tabular}

$* p<0.01$.

roughness of emery cloths stroked with the fingers grew with a Stevens exponent of 1.5 with respect to the diameter of the grit particles [16], that subjective hardness produced when rubber samples are squeezed between thumb and finger grew with a Stevens exponent of 0.8 with respect to physical hardness, and that perceived auditory roughness can be described by a power function with exponents ranging from 0.8 to 1.8 with an average value of 1.4 [33].

In the present work the perceived unpleasantness and roughness of amplitude-modulated steering wheel acceleration stimuli were found to depend on the psychophysical protocol used. As can be seen from Fig. 8, the growth exponent $n$ describing the human subjective response obtained by means of the Thurstone paired-comparison method was greater than unity for the perceived unpleasantness dimension and nearly equal to unity for the perceived roughness, whereas the exponents from the Borg CR-10 experiments remained nearly constant with a average value of 0.62 for the two dimensions. In this and other research studies performed by the present authors the use of the Borg CR-10 scale has led to smaller Stevens exponents than paired-comparison methods do. While difficult to demonstrate analytically, the lower exponent seems to be a reflection of an artefact in the Borg scale which occurs when only a portion of the dynamic range of the scale is used. In the research described here the mean subjective response of the test group was never greater than 5.0 , thus accounting for less than half the dynamic range of the Borg CR-10 scale.

Given the differences in the experimental data and the fitted Stevens power laws obtained by means of the two psychophysical protocols, consideration of related metrics is useful to lend support to one or the other of the data sets. An obvious metric for comparison is the VDV which is commonly used for quantifying the perceived intensity of the human response to vibration. Figure 9 presents the VDV, of the test stimuli as a function of $m^{*}=m-m_{\mathrm{th}}$. The VDVs ( $y$ axis) have been translated for comparison purposes by removing the sensory noise associated with the unmodulated sinusoidal signal $(m=0.0)$. From Fig. 9 it can be seen that the perceived intensity predicted by means of the VDV increases with increasing relative modulation index $m^{*}$ with a Stevens 


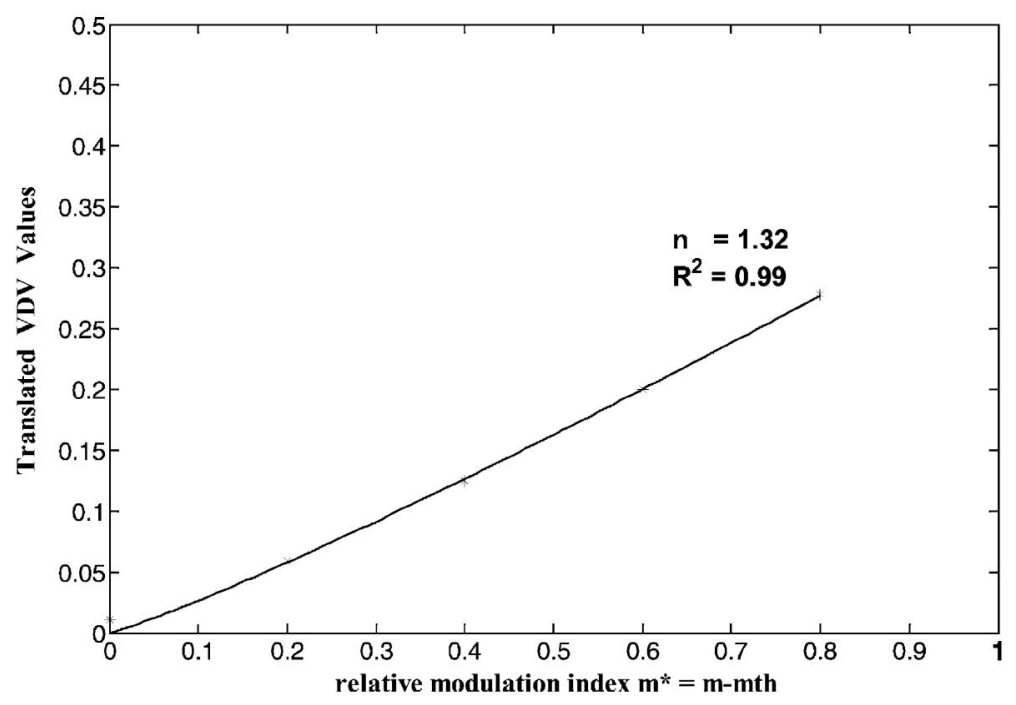

Fig. 9 Translated VDV as a function of relative modulation index $m^{*}=m-m_{\mathrm{th}}$

power exponent $n=1.32$. Since the VDV has found widespread acceptance in the human vibration and human testing communities, it is reasonable to assume that the power exponents obtained by means of Thurstone's paired-comparison method provide a closer measure of human response to amplitudemodulated steering wheel vibration than the results obtained using the Borg CR-10 scale.

\section{CONCLUSIONS}

The purpose of this study was to quantify the human subjective response to steering wheel vibration caused by diesel engine idle. Based on the results of previous investigations by the present authors, diesel engine idle vibration stimuli occurring at the steering wheel of automobiles was modelled as an amplitudemodulated harmonic signal having a carrier frequency of $26 \mathrm{~Hz}$, a modulation frequency of $6.5 \mathrm{~Hz}$ and an r.m.s. acceleration of $0.41 \mathrm{~m} / \mathrm{s}^{2}$. Evaluations of seven levels of modulation depth $m(0.0,0.1,0.2$, $0.4,0.6,0.8$, and 1.0) were performed in order to define the growth function of the human perceived disturbance. Two semantic descriptors of the human response and two psychophysical test protocols were used. The semantic attributes of unpleasantness and roughness were chosen as descriptors of the human response to idle vibration exposure, and the human subjective evaluations were performed by means of the Thurstone paired-comparison method (case III) and by means of the category ratio Borg CR-10 scale. The results suggest that there is a critical value of modulation depth $m=0.2$ below which human subjects cannot perceive differences in amplitude modulation and above which the stimulus-response relationship increases monotonically with a power function. The Thurstone paired-comparison indirect scaling method appears to be more effective in assessing amplitude-modulated steering wheel vibration than direct evaluation using the Borg CR-10 scale. The Stevens power exponents suggest that the perceived unpleasantness is non-linearly dependent on modulation depth $m$ with an exponent greater than 1 and that the perceived roughness is dependent with an exponent close to unity.

\section{ACKNOWLEDGEMENTS}

The authors would like to express their gratitude to Dr Gautam Kalghatgi and to Shell Global Solutions UK for their sponsorship and support.

\section{REFERENCES}

1 Dalpiaz, G., Rivola, A. and Rubini, R. Effectiveness and sensitivity of vibration processing techniques for local fault detection in gears. Mech. System Signal Processing, 2000, 14(3), 387-412.

2 Ajovalasit, M. and Giacomin, J. Analysis of variations in diesel engine idle vibration. Proc. Instn Mech. Engrs, Part D: J. Automobile Engineering, 2003, 217, 921-933.

3 Demers, M. A. Steering wheel vibration diagnosis. SAE paper 011607, 2001.

4 Hinze, P. C. and Cheng, W. K. Assessing the factors affecting SI engine cycle-to-cycle variations at idle. In Proceedings of the 27th International Symposium on Combustion, 1988, Vols 1-2, pp. 2119-2125 (The Combustion Institute, Pittsburgh, Pennsylvania). 
5 Dixon, J., Rhodes, D. M. and Phillips, A. V. The generation of engine half orders by structural deformation. In Proceedings of IMechE Conference on Vehicle NVH and Refinement, 1994, paper C487/032/ 94, pp. 9-17 (Professional Engineering Publishing Limited, Bury St Edmunds and London).

6 Lichty, L. C. Combustion Engine Processes, 1967 (McGraw-Hill Book, New York).

7 Rahnejat, H. Multi-body Dynamics: Vehicles, Machines and Mechanisms, 1998 (Professional Engineering Publishing Limited, Bury St Edmunds and London).

8 Priede, T. Noise and vibration control of the internal combustion reciprocating engine. In Noise and Vibration Control Engineering, Principles and Applications (Ed. L. L. A. V. Bernarek), 1992, pp. 665-707 (John Wiley, New York).

9 Hoard, J. and Rehagen, L. Relating subjective idle quality to engine combustion. SAE paper 970035 , 1997, pp. $1-5$

10 Verrilo, R. T. Psychophysics of vibrotactile stimulation. J. Acoust. Soc. Am., 1985, 77(1), 225-232.

11 Morioka, M. Effect of contact location on vibration perception threshold in the glabrous skin of the human hand. In Proceedings of the 34th United Kingdom Group Meeting on Human Responses to Vibration, 22-24 September 1999 (Ford Motor Company, Dunton, Essex).

12 Gescheider, G. A., Bolanowski Jr, S. J., Verrillo, R. T., Arpajian, D. J. and Ryan, T. F. Vibrotactile intensity discrimination measured by three methods. J. Acoust. Soc. Am., 1990, 87(1), 330-338.

13 Bensmaïa, S. J. and Hollins, M. Complex tactile waveform discrimination. J. Acoust. Soc. Am., 2000, 108(3), 1236-1245.

14 Weisenberger, J. M. Sensitivity to amplitudemodulated vibrotactile signals. J. Acoust. Soc. Am., 1986, 80(6), 1707-1715.

15 Lamoré, P. J. J. Envelope detection of amplitudemodulated high-frequency sinusoidal signals by skin mechanoreceptors. J. Acoust. Soc. Am., 1986, 79(4), 1082-1085.

16 Stevens, S. S. and Harris, J. R. The scaling of subjective roughness and smoothness. J. Expl Psychol., 1962, 64(5), 489-494.

17 Franzén, 0. The dependence of vibrotactile threshold and magnitude functions on stimulation frequency and signal level. Scand. J. Psychol., 1969, 10, 289-297.

18 Stevens, S. S. A metric for the social consensus. Science, 1966, 151, 530-541.

19 Ekman, G. and Kûnnapas, T. A further study of direct and indirect scaling methods. Scand. J. Psychol., 1963, 4, 77-80.

20 Burros, R. H., A solution for case III of the law of comparative judgment. Psychometrika, 1954, 19(1), 57-64.

21 Borg, G. Borg's Perceived Exertion and Pain Scales, 1998 (Human Kinetics, Champaign, Illinois).

22 Giacomin, J., Shayaa, M. S., Dormegnie, E. and Richard, L. A frequency weighting for the evaluation of steering wheel rotational vibration. Int. J. Ind. Ergonomics, 2004 (in press).
23 Viemeister, N. F. Temporal modulation transfer functions based upon modulation thresholds. J. Acoust. Soc. Am., 1979, 66(5), 1364-1380.

24 Gescheider, G. A., Hoffman, K. E., Harrison, M. A., Travis, M. L. and Bolanowski, S. J. The effects of masking on vibrotactile temporal summation in the detection of sinusoidal and noise signals. J. Acoust. Soc. Am., 1994, 95(2), 1006-1016.

25 Miwa, T. Evaluation methods for vibration effect. Part 7. The vibration greatness of the pulses. Ind. Health, 1968, 7, 143-164.

26 BS 6841: 1987 Guide to Measurement and Evaluation of Human Exposure to Whole-body Mechanical Vibration and Shock (British Standards Institution, London)

27 Griffin, M. J. Handbook of Human Vibration, 1990 (Academic Press, London).

28 Torgerson, W. S. Theory and Method of Scaling, 1958 (John Wiley, New York).

29 Gilson, E. Q. and Baddeley, A. D. Tactile short-term memory. Q. J. Expl Psychol., 1969, 21, 180-184.

30 Reynolds, D. D. and Keith, R. H. Hand-arm vibration, Part 1: analytical model of the vibration response characteristics of the hand. J. Sound Vibr., 1977, 51(2), 237-253.

31 ISO 13091-1 Mechanical Vibration-Vibrotactile Perception Thresholds for the Assessment of Nerve Dysfunction, Part 1: Methods of Measurement at the Fingertips, 2001 (International Standardization Organization, Geneva).

32 Stevens, S. S. (Ed.) Psychophysics: Introduction to its Perceptual, Neural and Social Prospects, 1986 (Transaction Books, New Brunswick, New Jersey).

33 Guirao, M. and Garavilla, J. M. Perceived roughness of amplitude-modulated tones and noise. J. Acoust. Soc. Am., 1976, 60(6), 1335-1338.

\section{APPENDIX}

\section{Notation}

$a \quad$ acceleration $\left(\mathrm{m} / \mathrm{s}^{2}\right)$

$A(t) \quad$ instantaneous amplitude of modulated signal $\left(\mathrm{m} / \mathrm{s}^{2}\right)$

CR category ratio

$f_{\mathrm{c}} \quad$ carrier frequency $(\mathrm{Hz})$

$f_{\mathrm{m}} \quad$ modulation frequency $(\mathrm{Hz})$

$\mathrm{H}_{i} \quad$ engine harmonic (order)

$m$ modulation depth

$m^{*} \quad$ modulation index equal to $m-m_{\text {th }}$

$m_{\text {th }} \quad$ amplitude modulation detection threshold

MTF modulation transfer function

$p \quad$ significance level

PSD power spectral density

SD standard deviation

VDV vibration dose value $\left(\mathrm{m} / \mathrm{s}^{1.75}\right)$ 